\title{
A Novel Non-Pharmaceutical Treatment for Patients with Mild Cognitive Impairment
}

\author{
Christina Darviri1 ${ }^{*}$, Chrysa Zavitsanou${ }^{1}$, Aikaterini Delikou1, Aikaterini Giotaki' ${ }^{1}$, \\ Artemios Artemiadis ${ }^{1}$, Aspasia Terentiou ${ }^{2}$, George P. Chrousos ${ }^{1,3}$ \\ ${ }^{1}$ Postgraduate Course Science of Stress and Health Promotion, School of Medicine, \\ University of Athens, Athens, Greece \\ ${ }^{2}$ Department of Neurology 417 NIMTS, Athens, Greece \\ ${ }^{3}$ First Department of Pediatrics, Children's Hospital Aghia Sofia, School of Medicine, \\ University of Athens, Athens, Greece \\ Email: "cdarviri@yahoo.com
}

Received 8 March 2016; accepted 13 May 2016; published 16 May 2016

Copyright (C) 2016 by authors and Scientific Research Publishing Inc.

This work is licensed under the Creative Commons Attribution International License (CC BY). http://creativecommons.org/licenses/by/4.0/

(c) (i) Open Access

\begin{abstract}
Mild cognitive impairment (MCI) represents a pre-dementia stage. Currently, there is no evidence for long-term efficacy of pharmacological treatment for preventing conversion to clinical dementia. Healthy lifestyle modifications, cognitive training and psychosocial initiatives are strongly recommended. This was an 8-week parallel randomized two-armed study. Eligible patients were randomized to intervention and control group, with the intervention group receiving the PSAI intervention including cognitive training, healthy lifestyle and stress management. Standardized questionnaires were used pre- and post-intervention. The between group analysis revealed a significant improvement in cognitive aspects, depression, stress, anxiety and self-efficacy post-intervention $(p<0.05, r>0.5)$. Nine patients in the intervention group had one point increase in the MMSE score and one patient had a two-point increment post-intervention. Future studies should expand these preliminary findings to larger samples, neuroimaging measurements and longer follow-up in order to ascertain PSAI's role in preventing conversion to clinical dementia.
\end{abstract}

\section{Keywords}

Mild Cognitive Impairment, Dementia, Treatment, Alzheimer, Intervention

\section{Introduction}

Mild cognitive impairment (MCI) is defined as the symptomatic pre-dementia stage on the continuum of cogni${ }^{*}$ Corresponding author.

How to cite this paper: Darviri, C., Zavitsanou, C., Delikou, A., Giotaki, A., Artemiadis, A., Terentiou, A., \& Chrousos, G. P. (2016). A Novel Non-Pharmaceutical Treatment for Patients with Mild Cognitive Impairment. Psychology, 7, 678-686. 
tive decline, characterized by subjective and objective impairment in cognition greater than that expected for the individual's age and educational level that is not severe enough to interfere with daily functioning (Petersen et al., 1999). The prevalence of MCI in adults aged 65 years and older is $10 \%-20 \%$; increasing with age (Langa \& Levine, 2014). There are two main clinical subcategories of MCI: amnestic MCI and non-amnestic MCI (Gauthier et al., 2006). MCI, especially amnestic, heralds the future onset of dementias, mainly Alzheimer's disease, but also other dementive diseases such as frontotemporal dementias, Lewy body disease, vascular dementia etc (Gauthier et al., 2006). The annual conversion rate in clinical significant dementia ranges from 5\% to 20\% depending on the population studied (Langa \& Levine, 2014).

Factors that increase the risk of cognitive impairment and MCI are cerebrovascular health factors (e.g. low compliance to Mediterranean diet and low folic acid intake), metabolic syndrome, prediabetes and diabetes, depression and polypharmacy (Langa \& Levine, 2014; Gauthier et al., 2006; Cooper et al., 2015). So far, no targeted medications (e.g. cholinesterase inhibitors) have been proven effective for preventing conversion to clinical dementia; drug treatments and non-pharmaceutical interventions are primarily aiming at reducing cerebrovascular risk factors (Gauthier et al., 2006). As a result, "healthy ageing” meaning healthy lifestyle (i.e. Mediterranean diet, systematic aerobic exercise and smoking cessation) along with mental activity and social engagement is currently the mainstay for MCI treatment and dementia prevention (Vega, 2014; Singh et al., 2014; Miller et al., 2012; Simon et al., 2012).

There is now ongoing and evolving research of several structured interventions that target improvement of cognition and/or stress reduction. In a relatively recent systematic review, multidisciplinary interventions using restorative and/or compensatory cognitive strategies have been proposed as useful for global cognitive functioning, especially memory but presumably also for mood, emotional aspects of cognitive dysfunction, daily life activities and quality of life in MCI patients (Simon et al., 2012). Moreover, as evidenced in another meta-analytic review psychological approaches (e.g. cognitive behavioral therapy, interpersonal therapy and counseling) added to usual care may reduce symptoms of depression and anxiety for people with MCI (Orgeta et al., 2014). It is also suggested that meditative stress management interventions (e.g. mindfulness, transcendental meditation, yoga etc) can exert some beneficial effects on cognition through several mechanisms such as providing protection from the cardiovascular risk factors of dementia, increasing brain perfusion, inducing neuroprotection and decreasing stress (for a review see Marciniak et al., 2014). Of note, cortisol, a stress hormone, detrimentally affects highly vulnerable and plastic brain areas such as prefrontal cortex and hippocampal functions and higher cortisol has been associated with impairments in executive function, and memory (Franz et al., 2011; McEwen \& Sapolsky, 1995).

In the era of functional imaging (i.e. fMRI), newly recognized neural circuits have been proposed to affect stress response and cognition. Default-mode-network (DMN) during resting state fMRI represents a functional conduit (comprised of the following: ventral medial prefrontal cortex, posterior cingulate cortex, dorsal medial prefrontal cortex, inferior parietal lobule and hippocampal formation) for stress relief, memory consolidation, creativity and "thought settlement" (collectively recognized as a meta-cognitive processes) (Whitfield-Gabrieli \& Ford, 2012). DMN activation is accompanied by perceptual decoupling allowing information derived mainly from memory to access the fronto-parietal network of attention and consciousness (Smallwood et al., 2012). In general, connectivity and task-dependent deactivation of DMN are progressively decreased with ageing. This phenomenon is perpetuated in MCI and Alzheimer's disease (Hafkemeijer et al., 2012). DMN function is increasingly impaired with growing age, which in its turn is associated with higher deposition of the $\beta$-amyloid protein in the brain (Ouchi \& Kikuchi, 2012). Interestingly, in a small pilot randomized controlled trial in MCI patients, an 8-week Mindfulness Based Stress Reduction (MBSR) program resulted in strengthening of the DMN connectivity and a trend towards less hippocampal atrophy (Wells \& Yeh, 2013).

Collectively, healthy lifestyle, stress management and cognitive rehabilitation are, until now, the basic elements of a successful management of MCI. In the light of this realization, along with the preliminary data on DMN and cognition, we have improvised a new non-pharmaceutical self-referring intervention called Pythagorean Self-Awareness Intervention (PSAI). The basis of PSAI is the Pythagorean philosophy which was modulated to conform to the aforementioned elements of MCI treatment. The aim of the current study was to ascertain the possible benefits of PSAI. Benefits were sought within cognitive and psychological indices of a small sample of MCI patients. This is a concept of thought study which intends to pave the way for neuroimaging studies (i.e. fMRI, volumetric MRI) by our team. 


\section{Method}

This is a non-blind, randomized two-armed parallel group (1:1 allocation) experimental study. No protocol change was made after study commencement.

The study was conducted at the outpatient neurological clinic of NIMTS hospital in Athens Greece from April to July 2014. The study protocol was approved by the Hospital's Scientific and Ethics Committee and was consistent with the Declaration of Helsinki. All eligible participants gave their written informed consent before study entry. All patients had the diagnosis of amnestic MCI based on 1) a subjective and/or objective memory complaint; 2) a Mini Mental Status Examination (MMSE) scores of 24 - 29; and 3) preservation of activities of daily living ascertained by the patients' or their relatives’ reports. Inclusion criteria were: age above 55 years old and residency in Athens. Exclusion criteria were: inability to read and write, psychosis, substance abuse and other organic disease that could impair cognitive function, participating in psychotherapeutic or other stress management therapies. Eligible patients were randomized to two groups; intervention and control group, using random numbers generated by an online generator (www.random.org). Follow-up period was eight weeks and both patients and researchers remained non-blind to assignment and measurements.

At baseline both groups received verbal and written information about the value of cognitive training, healthy lifestyle and stress management in MCI. Patients in the intervention group were administered the PSAI intervention comprising of 9 weekly sessions. During the first individualized session (day 0), patients were assessed by standardized questionnaires (see below) and a detailed discussion about cognitive training, healthy lifestyle (issues on smoking, diet, physical exercise and sleep) and stress management was made by an experienced psychologist. Pedometers were also given as an incentive for exercising. In the second session 3 - 5 patients participated in a biofeedback-assisted diaphragmatic breathing training and PSAI. Also guidance on diet, physical exercise and sleep was delivered. The third session was evaluative and patients discussed in groups practical issues or ideas that might had emerge during the last days. In the fourth group session participants were instructed more on the PSAI technique. All participants were asked to perform the technique two times per day (morning and bedtime) after 5 minutes of diaphragmatic breathing. In the next four group sessions lectures about memory, lifestyle and intrapersonal relationships were given, along with proper expatiation on the Pythagorean philosophy. During the final visit discussion about the technique and final assessments were made.

Patients in the control group were being telephoned in weekly intervals. In each communication patients were asked about their physical and psychological status with no intention for an in-depth counseling or intervention.

\subsection{PSAI}

PSAI technique was practiced twice per day (in the morning and at bedtime) in a quiet place while sitting comfortably in a chair. The content of the technique is based on the golden verses of Pythagoras

(http://en.wikipedia.org/wiki/The_golden_verses_of_Pythagoras). At night each individual had to follow three cognitive processes. Firstly, the individual had to recall every event through the day in the exact time sequence that it happened. In order to facilitate recall, events were categorized as follows: diet (e.g. "What did I eat or drink for breakfast” etc), exercise (scheduled or not), sleep (e.g. timing, hours of sleep etc.) and personal contacts. In the second step, the individual was asked to choose these events that seemed important to him/her and remember all the details accompanying them (e.g. the exact quantity of food at breakfast, all the words used in a discussion). For the categories of diet, exercise and sleep details were straightforward. However, for personal contacts the individual had to choose those that had seemed to bear any personal or emotional significance. During the last step each selected event was subjected to the critical appraisal using three questions: "In what have I done wrong? What have I done right? What have I omitted that I ought to have done?”. In this step the individual tried to remain detached by the emotional burden of the event and contemplate on the performed actions as if he/she was a "judge" of him-/herself. With regards to diet, exercise and sleep guidance had been already given during session one. Events or choices pertinent to personal relationships were judged freely by the individual, since the primary goal was to enhance self-awareness and not to criticize them. In the morning, each participant was instructed to recapitulate the results of the night practice and not to repeat the procedure.

\subsection{Measurements}

Sample characteristics: age, gender, education (primary/secondary vs. tertiary). 
Healthy Lifestyle: It was assessed by using the Healthy Lifestyle and Personal Control (HLPCQ) questionnaire (Darviri et al., 2014). It entails 26 items answered in a Likert-type scale (from $1=$ rarely to $4=$ always). An increased score denotes healthier lifestyle in terms of diet, daily routine, exercise, social support and positive thoughts.

Stress, Anxiety and Depression: They were measured using the corresponding 7 items of the Depression Anxiety Stress 21-item Scale (DASS-21) (Lovibond \& Lovibond, 1995). The responders declare the frequency of their symptoms in a Likert-type scale (from $0=$ did not apply to me at all to $3=$ applied to me very much, or most of the time) during the past week. Scores are produced by summing up all items. Higher scores indicate higher level of stress, anxiety or depression. The scale has been adapted in the Greek population (Lyrakos et al., 2011).

Geriatric Depression: It was assessed with the short form of Geriatric Depression Scale (GDS), a 15-item instrument, in which participants are asked to respond by answering yes or no in reference to how they felt over the past week (Yesavage \& Sheikh, 1986). Higher scores indicate higher depression. The tool has been validated in the Greek population (Fountoulakis et al., 1999).

Health locus of control: Health locus of control was measured using the 18-item Health Locus of Control Scale (HLC) developed by Wallston \& colleagues (1978). The respondents expressed their level of agreement to 18 statements in a 6-point Likert type scale (from 1 = strongly disagree to $6=$ strongly agree). The scale is built upon three 6-item subscales, namely: “internal health locus of control”, “external health locus of control” and “chance”. After summing up answers for each subscale, higher scores indicate higher strength of each type of health belief (total score range 6 - 36 for each subscale). HLC measurements were made at baseline and at the end of the 8 weeks. The instrument has been standardized for Greek populations (Karademas, 2009).

Self-efficacy: General Self-Efficacy Scale (GSES) was developed by Zhang \& Schwarzer (1995). This is a self-report questionnaire designed to measure self-confidence in dealing with various situations such as stress, novel experiences etc. Items were rated in 4-point Likert-type scale from 1 (not true at all) to 4 (very true).

Mini Mental Status Examination: The MMSE was developed by Folstein (1975) and is commonly used to examine the cognitive function of older adults. The MMSE contains 30 items, and each item is assigned a binary score ( 1 for a correct answer and 0 otherwise). The highest possible score is 30 .

Five Word Test (FWT): Dubois' five-word test is an episodic memory test where subjects are asked to learn five words of five different semantic groups; it includes immediate free recall exercises and postponed free recall activities (Dubois et al., 2002). In the case of recall difficulty, a cue is suggested.

Symbol Digit Modalities Test (SDMT): This test is widely used for evaluation of the information processing speed (Sheridan et al., 2006). It consists of nine symbols which correspond to nine numbers (from 1 to 9). The responder was presented with a set of pseudo-randomized sequence of symbols and asked to indicate by voice the corresponding numbers in 90 seconds. The score indicates the number of correct responses during this time period.

Brief Visuospatial Memory Test-Revised (BVMT-R): This test evaluates visuospatial memory (Benedict, 1997). The individual was presented with a matrix of six items in two columns and three rows for ten seconds and he/she was asked to replicate it unaided using pencil and paper, taking as much time needed. The process was repeated three times. Each drawing was evaluated for both its placement (1 point) and its accuracy (1 point). Thus the maximum score yielded by the three trials was 36, indicating unimpaired visuospatial direct memory recall.

\subsection{Statistical Analyses}

Baseline characteristics were compared between groups by using the non-parametric Mann-Whitney U test and exact chi-square for categorical variables. Mann-Whitney U test was also used for the comparison of the outcomes' differences (value at the end of the follow-up minus baseline value). Effect sizes rho were also calculated according to the formula $r=\mathrm{Z} / \mathrm{N} 1 / 2$ where rhos $0.1,0.3$ and 0.5 denote small, moderate and large effect sizes, respectively. The level of significance was set at $p<0.05$. Statistical analyses were done using SPSS 21.0 software (SPSS Inc., Chicago, IL, USA).

\section{Results}

In total, 141 patients were assessed for study entry, 89.4\% of which were found eligible according to the inclu- 
sion/exclusion criteria (Figure 1). Of the 126 eligible patients 67 (53.2\%) refused to participate. The main reasons for refusal were the long follow-up period and the disbelief in non-pharmaceutical interventions for treating MCI. As a result 59 patients were equally randomized to the two study groups. The drop-out rate was similar in the two study groups (intervention group 40.0\% vs. control group 37.9\%). Reasons are presented in Figure 1.

Table 1 presents baseline group comparisons. The majority of the patients were women with a mean age of approximately 72 years old. In general, no significant group differences were noted between the intervention group and the control group.

Table 2 presents group comparisons for the differences (last visit value minus baseline value) for the main study measurements. Beneficial outcomes were found nearly for all outcomes except for chance HLC. As seen in Table 2. The control group had no change between baseline and last visit values for nearly all outcomes. According to rho values all significant differences were of large effect size except for healthy lifestyle, external HLC which showed moderate change. We should also report that nine of the MCI patients in the intervention

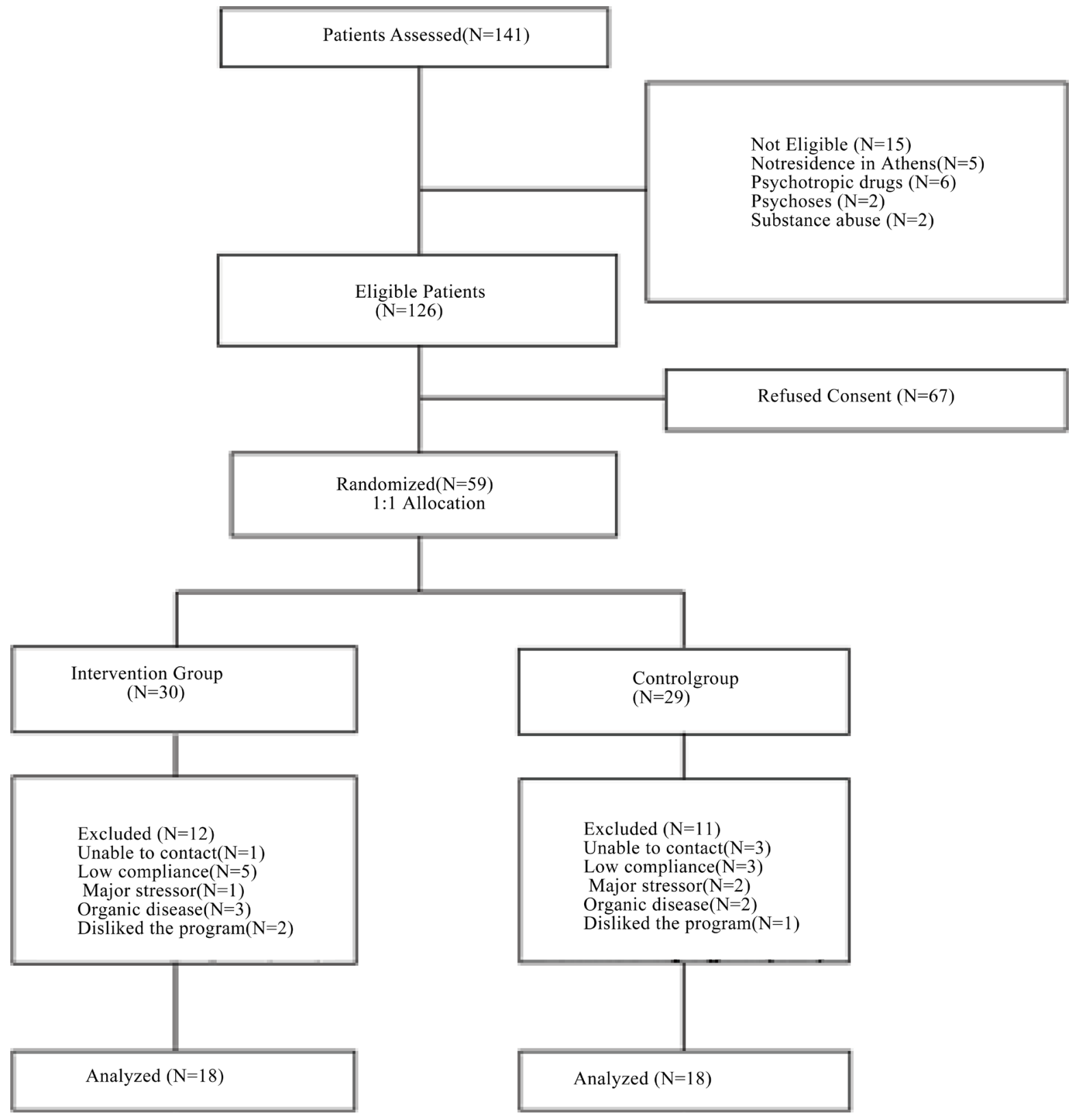

Figure 1. Flowchart of the study. 
Table 1. Baseline sociodemographic and outcome characteristics.

\begin{tabular}{cccc}
\hline & Intervention Group (N = 18) & Control Group (N = 18) & $p$ Value \\
\hline Females (\%) & $13(72.2 \%)$ & $14(77.8 \%)$ & 1.00 \\
Mean Age (Min-Max) & $71.94(57-82)$ & $71.55(63-82)$ & 0.72 \\
Primary/Secondary Education (\%) & $14(77.8 \%)$ & $12(66.7 \%)$ & 0.71 \\
Mean HLPCQ (Min-Max) & $38.88(26-52)$ & $40.61(26-52)$ & 0.382 \\
Mean Stress (Min-Max) & $8.27(0-14)$ & $8.66(4-17)$ & 0.67 \\
Mean Anxiety (Min-Max) & $4.05(1-9)$ & $6.11(0-18)$ & 0.17 \\
Mean Depression (Min-Max) & $5.44(0-15)$ & $5.50(0-14)$ & 0.87 \\
Mean GDS (Min-Max) & $6.94(0-12)$ & $6.94(0-12)$ & 0.98 \\
Mean Internal HLC (Min-Max) & $23.88(17-35)$ & $24.05(17-30)$ & 0.81 \\
Mean Chance HLC (Min-Max) & $17.22(9-31)$ & $20.22(8-30)$ & 0.15 \\
Mean External HLC (Min-Max) & $24.55(18-36)$ & $24.05(14-32)$ & 0.49 \\
Mean Self-efficacy (Min-Max) & $29.55(16-37)$ & $28.27(16-36)$ & 0.42 \\
Mean FWT (Min-Max) & $14.77(9-26)$ & $12.77(8-17)$ & 0.32 \\
Mean MMSE (Min-Max) & $26.55(24-29)$ & $25.77(24-29)$ & 0.23 \\
Mean SDMT (Min-Max) & $21.83(7-44)$ & $19.33(8-32)$ & 0.45 \\
Mean BVMT-R (Min-Max) & $10.00(5-22)$ & $7.55(5-20)$ & 0.27 \\
\hline
\end{tabular}

Min: Minimum value, Max: Maximum value, HLPCQ: Healthy Lifestyle and Personal Control Questionnaire, GDS: Geriatric Depression Scale, HLC: Health Locus of Control, FWT: Five Word Test, MMSE: Mini Mental Status Examination, SDMT: Symbol Digit Modality Test, BVRT: Brief Visuospatial Memory Test-Revised.

Table 2. Between group comparisons for the study’s outcomes.

\begin{tabular}{|c|c|c|c|c|}
\hline & Intervention Group ( $\mathrm{N}=18)$ & Control Group (N = 18) & $p$ Value & Rho Effect Size ${ }^{1}$ \\
\hline$\Delta$ Mean Healthy Lifestyle (Min-Max) & $3.94(-1-13)$ & $0(0-0)$ & $0.019^{*}$ & 0.39 \\
\hline$\Delta$ Mean Stress (Min-Max) & $-3.83(-8,6)$ & $0(0,0)$ & $<0.001^{*}$ & 0.77 \\
\hline$\Delta$ Mean Anxiety (Min-Max) & $-1.88(-5,2)$ & $0(0,0)$ & $<0.001^{*}$ & 0.69 \\
\hline$\Delta$ Mean Depression (Min-Max) & $-2.61(-7,2)$ & $0(0,0)$ & $<0.001^{*}$ & 0.73 \\
\hline$\Delta$ Mean GDS (Min-Max) & $-4.00(-8,1)$ & $0(0,0)$ & $<0.001^{*}$ & 0.73 \\
\hline$\Delta$ Mean Internal HLC (Min-Max) & $3.00(-11,11)$ & $0(0,0)$ & $<0.001^{*}$ & 0.69 \\
\hline$\Delta$ Mean Chance HLC (Min-Max) & $-2.27(-21,8)$ & $0(0,0)$ & 0.098 & 0.28 \\
\hline$\Delta$ Mean External HLC (Min-Max) & $-3.44(-15,6)$ & $0(0,0)$ & $0.042^{*}$ & 0.34 \\
\hline$\Delta$ Mean Self-efficacy (Min-Max) & $1.44(0,10)$ & $0.66(0,12)$ & $0.001^{*}$ & 0.53 \\
\hline$\Delta$ Mean FWT (Min-Max) & $2.00(0,5)$ & $0(0,0)$ & $<0.001^{*}$ & 0.68 \\
\hline$\Delta$ Mean MMSE (Min-Max) & $0.61(0,2)$ & $0(0,0)$ & $<0.001^{*}$ & 0.61 \\
\hline$\Delta$ Mean SDMT (Min-Max) & $2.38(-2,8)$ & $0(0,0)$ & $<0.001^{*}$ & 0.73 \\
\hline$\Delta$ Mean BVMT-R (Min-Max) & $1.94(-1,6)$ & $-0.05(-1,0)$ & $<0.001^{*}$ & 0.61 \\
\hline
\end{tabular}

${ }^{1} R=\sqrt{[Z / N]}, 0.1$ small, 0.3 moderate, 0.5 large effect size.

Min: Minimum value, Max: Maximum value, HLPCQ: Healthy Lifestyle and Personal Control Questionnaire, GDS: Geriatric Depression Scale, HLC: Health Locus of Control, FWT: Five Word Test, MMSE: Mini Mental Status Examination, SDMT: Symbol Digit Modality Test, BVRT: Brief Visuospatial Memory Test-Revised.

${ }^{*}$ Level of significance $p<0.05$.

group had one point increase in the MMSE score and one patient had a two-point increment at the end of the 8-week follow-up.

Finally, no side effects or serious practical issues during the intervention were reported.

\section{Discussion}

In this concept of thought study, our newly introduced intervention has been documented with beneficial findings for healthy lifestyle, psychological and cognitive indices in MCI patients. As stated in the introduction, 
PSAI is designed to address the three fundamental treatment approaches of MCI, meaning healthy lifestyle, stress management and cognitive rehabilitation. As such, our preliminary results validate the original idea of PSAI. During the study, some qualitative data are also gathered for patients in the intervention group; all MCI patients are favorable for the intervention reporting higher optimism, self-confidence, positive self-image, more sociability, physical exercise, healthier diet, positive thoughts, good mood, setting limits to others, better sleep and increase self-awareness at the end of the follow up. In the absence of previous PSAI studies, our further discussion will focus on the putative processes engaged in PSAI and their role in the healthy lifestyle decision making, emotional defusion and cognitive rehabilitation.

Introspection, emotion awareness and self-judgment are the cornerstones of PSAI but also the most elusive processes in terms of the contemporary neuroscience. The conception of the idea of the inner experience of thoughts and feelings straddles between philosophy and psychodynamic psychology crumpling against the principles of behaviorism. PSAI in its principle encourages a pristine inner experience process by imposing inner speaking and contemplation on daily actions and feelings. In an elegant fMRI study by Herwig et al., emotion introspection (self-awareness of emotions with no cognitive intention of regulating them) is associated with decreased activation of amygdala and increases activation of dorsal brain areas presumably indicating attenuation of the emotional arousal. In the same study, cognitive self-reflection ("think about yourself, reflect who you are, about your goals, etc.”) is accompanied by activation of ventral brain areas (i.e. medial and lateral prefrontal cortex) and especially of the left hemisphere, reflecting, as suggested by the authors, the implementation of self-regulation and inner speaking, respectively (Herwig et al., 2010). Interestingly, a recent review states that "using non-first-person pronouns and one's own name (rather than first-person pronouns) during introspection enhances self-distancing...” and that this may buffer distress allowing more adjustive thoughts, feelings and behaviors (Kross et al., 2014). We should note that in our study, except for the recorded psychological benefits, PSAI results also in better self-efficacy and health locus of control. Collectively, PSAI can ameliorate emotional distress and promote prudent decision making for healthy lifestyle and interpersonal behaviors.

In a first, assessment PSAI can be categorized in the meditation techniques, but we have several objections in such an approach. There are several types of meditation such as mindfulness, transcendental meditation, yoga etc. The infrastructure of these meditative techniques is characterized by the following features: 1) shift of attention from external to internal stimuli (e.g. thoughts, feelings, words, body sensations etc); 2) concentration on a pre-specified internal stimulus, ignoring other sensory or mental perturbations (Epel et al., 2009). Although the former also applies to PSAI, the latter does not. PSAI actually represents a meta-cognitive process meaning a conscious awareness of own's cognitive state and private monitoring of self-states. Memory has an integral role in this process since it provides all the necessary data recollections of events and previous experiences in order to facilitate robust self-judgment and decision making. PSAI, unlike mindfulness, encourages the individual to concentrate on certain daily nuisances recognized by their negative emotional burden that impose on the person, as such supposedly allowing a more targeted self-improvement process than mindfulness. Presumably this approach leads to stress defusion and cognitive reappraisal of stressors. In the long-term individuals may be able to dispense "automated" behaviors and thoughts that contribute to maladjusted and stressful cognitive schemas.

Humans, like other primates, are endowed with the capacity of cognitive plasticity based upon the nervous system's inherent ability to adapt its structural organization under environmental stress such as cellular damage (Greenwood \& Parasuraman, 2010). A measurable counterpart of this capacity is the cognitive reserve which, when high, it provides protection to developing dementia (Valenzuela \& Sachdev, 2006). In a relatively recent systematic review, multidisciplinary interventions using restorative and/or compensatory strategies have been suggested as beneficial for global cognitive functioning, especially memory but presumably also for mood, emotional aspects of cognitive dysfunction, daily life activities and quality of life in MCI patients (Simon et al., 2012). Episodic memory recall in PSAI could be deemed as a compensatory strategy, although not so sophisticated like for example visual imagery, cueing, mind mapping etc. Surprisingly, our results were indicative of benefits both in memory and other cognitive domains such as information processing speed. This finding may suggest that PSAI contributes to cognitive rehabilitation by mechanisms other than the simply recalling daily events. Stress management could be the major culprit as stated in the introduction part (for a review see Marciniak et al., 2014).

In addition we quote some qualitative data which derived from self-reported comments post-intervention. Those comments were coded and analyzed statistically. Specifically, there was: a) a $45 \%$ improvement in sleep quality; b) an $80 \%$ stated that PSAI resulted in more energy and self-empowerment; c) a $66 \%$ declared having a 
memory improvement; d) a $60 \%$ reported changes in dietary habits and weight loss; e) a 55\% stated that through PSAI was able to set boundaries and improve his/her interpersonal relationships; f) a 55\% implemented the advice given regarding physical exercise; g) a 50\% reported achieving a better mood regulation and anger management; and h) a 5\% did not experience any differences through PSAI.

We admit that the study had a number of limitations. Firstly, our sample was small thus our results cannot be generalized. Secondly, all measurement were self-report, as such there is always the chance of favorable to the intervention bias. Thirdly, we did not ascertain if benefits were maintained after the intervention. Fourthly, cognitive reserve was not taken into account. However, at least with respect to education our sample was quite homogenous. Finally, follow-up was short and no calculation of the conversion rate to clinical significant dementia was made.

Conclusively, PSAI is a newly introduced non-pharmaceutical intervention that showed benefits for healthy lifestyle, stress management and cognitive rehabilitation in MCI patients. Future studies should expand our findings to larger samples, neuroimaging measurements and longer follow-up in order to ascertain PSAI's role in preventing conversion to clinical dementia.

\section{Acknowledgements}

We would like to thank the philosopher Dr. Altani for inspiring us to develop and design PSAI in adherence to the basic principles of the Pythagorean philosophy.

\section{References}

Benedict, R. (1997). Brief Visuospatial Memory Test-Revised: Professional Manual. Odessa, FL: Psychological Assessment Resources.

Cooper, C., Sommerlad, A., Lyketsos, C.G., \& Livingston, G. (2015). Modifiable Predictors of Dementia in Mild Cognitive Impairment: A Systematic Review and Meta-Analysis. The American Journal of Psychiatry, 172, 323-334.

http://dx.doi.org/10.1176/appi.ajp.2014.14070878

Darviri, C., Alexopoulos, E.C., Artemiadis, A.K., Tigani, X., Kraniotou, C., Darvyri, P., \& Chrousos, G.P. (2014). The Healthy Lifestyle and Personal Control Questionnaire (HLPCQ): A Novel Tool for Assessing Self-Empowerment through a Constellation of Daily Activities. BMC Public Health, 14, 995. http://dx.doi.org/10.1186/1471-2458-14-995

Dubois, B., Touchon, J., Portet, F., Ousset, P.J., Vellas, B., \& Michel, B. (2002). The “5 Words”: A Simple and Sensitive Test for the Diagnosis of Alzheimer's Disease. La Presse Médicale, 31, 1696-1699.

Epel, E., Daubenmier, J., Moskowitz, J.T., Folkman, S., \& Blackburn, E. (2009). Can Meditation Slow Rate of Cellular Aging? Cognitive Stress, Mindfulness, and Telomeres. Annals of the New York Academy of Sciences, 1172, 34-53. http://dx.doi.org/10.1111/j.1749-6632.2009.04414.x

Folstein, M.F., Folstein, S.E., \& McHugh, P.R. (1975). “Mini-Mental State”. A Practical Method for Grading the Cognitive State of Patients for the Clinician. Journal of Psychiatric Research, 12, 189-198. http://dx.doi.org/10.1016/0022-3956(75)90026-6

Fountoulakis, K.N., Tsolaki, M., Iacovides, A., Yesavage, J., O’Hara, R., Kazis, A., \& Ierodiakonou, Ch. (1999). The Validation of the Short Form of Geriatric Depression Scale (GDS) in Greece Published in Aging. Clinical and Experimental Research, 11, 367-372.

Franz, C.E., O’Brien, R.C., Hauger, R.L., Mendoza, S.P., Panizzon, M.S., Prom-Wormley, E., \& Kremen, W.S. (2011). Cross-Sectional and 35-Year Longitudinal Assessment of Salivary Cortisol and Cognitive Functioning: The Vietnam Era Twin Study of Aging. Psychoneuroendocrinology, 36, 1040-1052. http://dx.doi.org/10.1016/j.psyneuen.2011.01.002

Gauthier, S., Reisberg, B., Zaudig, M., Petersen, R.C., Ritchie, K., Broich, K., \& Winblad, B. (2006). International Psychogeriatric Association Expert Conference on Mild Cognitive Impairment. The Lancet, 367, 1262-1270.

Greenwood, P.M., \& Parasuraman, R. (2010). Neuronal and Cognitive Plasticity: A Neurocognitive Framework for Ameliorating Cognitive Aging. Frontiers in Aging Neuroscience, 2, 1-14. http://dx.doi.org/10.3389/fnagi.2010.00150

Hafkemeijer, A., van der Grond, J., \& Rombouts, S. A. (2012). Imaging the Default Mode Network in Aging and Dementia. Biochimica et Biophysica Acta (BBA)-Molecular Basis of Disease, 1822, 431-441. http://dx.doi.org/10.1016/j.bbadis.2011.07.008

Herwig, U., Kaffenberger, T., Jäncke, L., \& Brühl, A. B. (2010). Self-Related Awareness and Emotion Regulation. NeuroImage, 50, 734-741. http://dx.doi.org/10.1016/j.neuroimage.2009.12.089

Karademas, E. C. (2009). Effects of Exposure to the Suffering of Unknown Persons on Health-Related Cognitions, and the Role of Mood. Health, 13, 491-504. http://dx.doi.org/10.1177/1363459308336793 
Kross, E., Bruehlman-Senecal, E., Park, J., Burson, A., Dougherty, A., Shablack, H. et al. (2014). Self-Talk as a Regulatory Mechanism: How You Do It Matters. Journal of Personality and Social Psychology, 106, 304-324. http://dx.doi.org/10.1037/a0035173

Langa, K. M., \& Levine, D. A. (2014). The Diagnosis and Management of Mild Cognitive Impairment: A Clinical Review. JAMA, 312, 2551-2561. http://dx.doi.org/10.1001/jama.2014.13806

Lovibond, S. H., \& Lovibond, P. (1995). Manual for the Depression Anxiety Stress Scales (2nd ed.). Sydney: Psychology Foundation.

Lyrakos, G. N., Arvaniti, C., Smyrnioti, M., \& Kostopanagiotou, G. (2011). Translation and Validation Study of the Depression Anxiety Stress Scale in the Greek General Population and in a Psychiatric Patient's Sample. European Psychiatry, 26, 1731. http://dx.doi.org/10.1016/S0924-9338(11)73435-6

Marciniak, R., Sheardova, K., Cermáková, P., Hudeček, D., Sumec, R., \& Hort, J. (2014). Effect of Meditation on Cognitive Functions in Context of Aging and Neurodegenerative Diseases. Frontiers in Behavioral Neuroscience, 8, 17. http://dx.doi.org/10.3389/fnbeh.2014.00017

McEwen, B. S., \& Sapolsky, R. M. (1995). Stress and Cognitive Function. Current Opinion in Neurobiology, 5, $205-216$. http://dx.doi.org/10.1016/0959-4388(95)80028-x

Miller, D. I., Taler, V., Davidson, P. S. R., \& Messier, C. (2012). Measuring the Impact of Exercise on Cognitive Aging: Methodological Issues. Neurobiology of Aging, 33, 622.e29-622.e43.

http://dx.doi.org/10.1016/j.neurobiolaging.2011.02.020

Orgeta, V., Qazi, A., Spector, A. E., \& Orrell, M. (2014). Psychological Treatments for Depression and Anxiety in Dementia and Mild Cognitive Impairment. Cochrane Database of Systematic Reviews, No. 1, Article No. CD009125. http://dx.doi.org/10.1002/14651858.cd009125.pub2

Ouchi, Y., \& Kikuchi, M. (2012). A Review of the Default Mode Network in Aging and Dementia Based on Molecular Imaging. Reviews in the Neurosciences, 23, 263-268. http://dx.doi.org/10.1515/revneuro-2012-0029

Petersen, R. C., Smith, G. E., Waring, S. C., Ivnik, R. J., Tangalos, E. G., \& Kokmen, E. (1999). Mild Cognitive Impairment: Clinical Characterization and Outcome. Archives of Neurology, 56, 303-308.

http://dx.doi.org/10.1001/archneur.56.3.303

Sheridan, L. K., Fitzgeralda, H. E., Adams, K. M., Nigga, J. T., Martel, M. M., Puttler, L. et al. (2006). Normative Symbol Digit Modalities Test Performance in a Community-Based Sample. Archives of Clinical Neuropsychology, 21, 23-28. http://dx.doi.org/10.1016/j.acn.2005.07.003

Simon, S. S., Yokomizo, J. E., \& Bottino, C. M. C. (2012). Cognitive Intervention in Amnestic Mild Cognitive Impairment: A Systematic Review. Neuroscience \& Biobehavioral Reviews, 36, 1163-1178. http://dx.doi.org/10.1016/j.neubiorev.2012.01.007

Singh, B., Parsaik, A. K., Mielke, M. M., Erwin, P. J., Knopman, D. S., Petersen, R. C., \& Roberts, R. O. (2014). Association of Mediterranean Diet with Mild Cognitive Impairment and Alzheimer's Disease: A Systematic Review and MetaAnalysis. Journal of Alzheimer's Disease, 39, 271-282.

Smallwood, J., Brown, K., Baird, B., \& Schooler, J. W. (2012). Cooperation between the Default Mode Network and the Frontal-Parietal Network in the Production of an Internal Train of Thought. Brain Research, 1428, 60-70.

http://dx.doi.org/10.1016/j.brainres.2011.03.072

Valenzuela, M. J., \& Sachdev, P. (2006). Brain Reserve and Dementia: A Systematic Review. Psychological Medicine, 36, 441-454. http://dx.doi.org/10.1017/S0033291705006264

Vega, J. N., \& Newhouse, P. A. (2014). Mild Cognitive Impairment: Diagnosis, Longitudinal Course, and Emerging Treatments. Current Psychiatry Reports, 16, 490. http://dx.doi.org/10.1007/s11920-014-0490-8

Wallston, K. A., Wallston, B. S., \& DeVellis, R. (1978). Development of the Multidimensional Health Locus of Control Scale. Health Education \& Monographs, 6, 160-170. http://dx.doi.org/10.1177/109019817800600107

Wells, R. E., Yeh, G. Y., Kerr, C. E., Wolkin, J., Davis, R. B., Tan, Y. et al. (2013). Meditation’s Impact on Default Mode Network \& Hippocampus in Mild Cognitive Impairment: A Pilot Study. Neuroscience Letters, 556, 15-19. http://dx.doi.org/10.1016/j.neulet.2013.10.001

Whitfield-Gabrieli, S., \& Ford, J. M. (2012). Default Mode Network Activity and Connectivity in Psychopathology. Annual Review of Clinical Psychology, 8, 49-76. http://dx.doi.org/10.1146/annurev-clinpsy-032511-143049

Yesavage, J. A., \& Sheikh, J. I. (1986). 9/Geriatric Depression Scale (GDS): Recent Evidence and Development of a Shorter Violence. Clinical Gerontologist, 5, 165-173. http://dx.doi.org/10.1300/J018v05n01_09

Zhang, J. X., \& Schwarzer, R. (1995). Measuring Optimistic Self-Beliefs: A Chinese Adaptation of the General Self-Efficacy Scale. Psychologia, 38, 174-181. 